\title{
Performance of pinning-controlled synchronization
}

\author{
Luiz Felipe R. Turci ${ }^{*}$ and Elbert E. N. Macau ${ }^{\dagger}$ \\ National Institute for Space Research - INPE, São José dos Campos, Brazil \\ (Received 9 November 2010; revised manuscript received 28 February 2011; published 15 July 2011)
}

\begin{abstract}
In this work we address the problem of controlling a complex network toward a synchronous evolution by using the pinning-control strategy. In considering this control approach, we investigate the effect of the number of pinned nodes and how the strategy performance behaves in accordance with the type of nodes that are chosen to be controlled. The roles of the control and coupling gains are also discussed.
\end{abstract}

DOI: 10.1103/PhysRevE.84.011120

PACS number(s): 05.45.Xt, 05.45.Gg

\section{INTRODUCTION}

Ordered collective motion is a phenomenon commonly observed in nature. One can find it in large populations of interconnected oscillators in physics (e.g., in arrays of superconducting Jesephson junctions [1]), biology (e.g., in neurons in the brain [2]), and chemistry (e.g., in arrays of electrochemical oscillators [3]). Also, ordered collective motion can be found in large populations of live organisms such as flocks of birds or schools of fish. The common feature in all of these cases is the scenario in which agents interact with each other by some sort of coupling so that the convergence to the organized behavior emerges from arbitrary, different initial conditions; moreover, this process is robust to small differences among individuals. Furthermore, the collective motion behaves as a single, virtual monolithic individual, so that the entire set can have its dynamics changed (e.g., changing its trajectory without losing the ordered characteristic) by acting on the dynamics of the constitutive elements. This paper is related to this last stated feature. As such, we aim to understand how it is possible to change with efficacy this ordered monolithic behavior by acting on the individual dynamics of the constitutive elements.

Recent works have shown that the scenario of collective motion can be reproduced in a set of identical (or almost identical) low-dimensional nonlinear oscillators coupled to each other through well-defined links [3-5]. This model can be described by a network with fixed topology in which the nodes are the oscillators and the coupling links are the edges [4]. As such, according to the way the individuals are coupled to each other, the resulting network is classified as a hierarchical, random, small-world, or scale-free network [5]. In this model, an ordered collective behavior is represented by the situation in which the oscillators are synchronized [6]. Several works consider this scenario to understand how ordered collective motion appears or to develop control strategies that can be applied to drive the network dynamics to ordered states.

In this regard, researchers using a network model are investigating how it is possible to change collective behaviors based on the action imposed over the dynamics of its individual elements (oscillators) [7]. In a limit situation, the collective dynamics can be properly changed if the control action is applied to all elements of the set. However, a more efficient and

\footnotetext{
*felipeturci@yahoo.com.br

†elbert@lac.inpe.br
}

realistic approach would be to take advantage of the robustness presented in the synchronization behavior to act just on some judiciously chosen elements of the network. Based on that, the elements in which the control strategy is not applied could end up changing their dynamics to preserve the synchronized state of the network. The main idea consists of controlling just a fraction of the network nodes by using some local feedback control applied to them. This approach is known as pinning control, which was discussed by Grigoriev et al. [7]. This control approach is illustrated in Fig. 1.

In this work we aim to determine guidelines for proper control, with efficacy, of the synchronized state of a network of chaotic oscillators using the pinning-control strategy. In this context, efficacy means that the desired changes of the synchronized behavior are to be accomplished by imposing the feedback control over a reduced number of nodes. The key point here is to find the more appropriate nodes over which the control action must be applied. They must be properly chosen to allow us to take advantage of the stability of the synchronized state. It is this stability that allows us to control just a reduced number of nodes in order to change the system dynamics while the network synchronization is kept throughout the changing process. As such, these guidelines might be dependent on the network topology and also on the degree of the nodes that are chosen to receive the action of the feedback control. These dependencies are also investigated and reported here. Actually, those issues can be associated with a network characteristic entitled pinning controllability, which was previously discussed in [8-11] but only considering statistical limits. However, here instead we have taken into account a finite population of individuals (oscillators) and have used intensive numerical simulations to achieve our results. The results are subsequently analyzed in the context of a mathematical model that allows us to explain them. Our main findings are the following: (1) Increasing the control gain $q$ and/or the set of controlled individual nodes does not always guarantee meaningful improvement of the synchronization performance. (2) High degree nodes are a good choice for pinning in order to improve synchronization performance. In addition, we compare these results with the ones presented in previously mentioned works $[10,11]$.

The paper is organized as follows: in Sec. II the general analytical result is presented, in Sec. III the numerical analysis on pinning synchronization performance is presented, and finally in Sec. IV conclusions are given based on the results obtained in this work. 


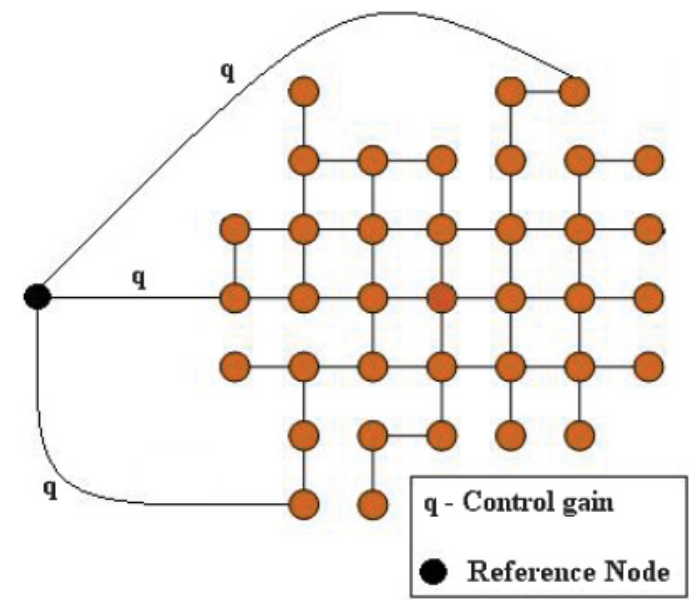

FIG. 1. (Color online) Pinning-control network synchronization strategy schematic in which a reference node acts on a small set of individuals of the complex network.

\section{COLLECTIVE SYSTEM MODEL AND ANALYTICAL ANALYSIS}

Let us consider a complex undirected network of identical nonlinear oscillators defined as follows:

$$
\dot{\mathbf{x}}_{i}(t)=\mathbf{f}\left[\mathbf{x}_{i}(t), t\right]-\sigma \sum_{j \in \mathcal{N}_{i}}\left[\mathbf{x}_{i}(t)-\mathbf{x}_{j}(t)\right], \quad i=1, \ldots, N,
$$

in which $i=1, \ldots, N ; \mathbf{x}_{i}(t)=\left[x_{i 1}(t), \ldots, x_{i n}(t)\right]^{T} \in \mathbb{R}^{n}$ is the state vector of the $i$ th node of the network, and $\mathbf{f}: \mathbb{R}^{n} \times$ $\mathbb{R}^{+} \rightarrow \mathbb{R}^{n}$ is a smooth nonlinear vector field. The parameter $\sigma$ is a unique global coupling strength among nodes assumed to be constant and time invariant. $\mathcal{N}_{i}$ is the set of neighbors of node $i$, that is, the set of nodes connected to node $i$. The set of all network connections is given by $\mathcal{E}$, and defined by the Laplacian matrix $L=\left[l_{i j}\right]$ as follows:

$$
l_{i j}= \begin{cases}-1 & \text { if }(i, j) \in \mathcal{E}, \\ -\sum_{k \in \mathcal{N}_{i}} l_{i k} & \text { if } i=j, \\ 0 & \text { otherwise, }\end{cases}
$$

with $l_{i j}=l_{j i}$, since only undirected networks are considered.

Given the node dynamics, it can be shown that for a network with diffusive coupling, in which all nodes' states are coupled as in Eq. (1), there is a critical value of $\sigma$ above which the network synchronizes [12]. ${ }^{1}$ The problem of finding this critical value of $\sigma$ is termed the synchronizability problem, and has been solved for linear (diffusive) coupling mainly by using the so-called master stability function approach (introduced in [13]). However, if one wants to synchronize the network onto a common synchronized state given by some external reference, say $\mathbf{x}_{s}(t)$, a strategy other than only adjusting $\sigma$ needs to be taken into account.

\footnotetext{
${ }^{1}$ A complex network with diffusive coupling, where all nodes states are coupled, has an unbounded synchronization region and consequently the synchronizability of the network is characterized by the eigenvalue of the Laplacian matrix with the lowest nonzero real part [12].
}

Given a complex network represented by Eq. (1), the application of pinning control results in the following:

$$
\begin{gathered}
\dot{\mathbf{x}}_{i}(t)=\mathbf{f}\left[\mathbf{x}_{i}(t), t\right]-\sigma \sum_{j \in \mathcal{N}_{i}}\left[\mathbf{x}_{i}(t)-\mathbf{x}_{j}(t)\right]-\delta_{i} q_{i}\left[\mathbf{x}_{i}(t)-\mathbf{x}_{s}(t)\right], \\
i=1, \ldots, N .
\end{gathered}
$$

Here, the function $\delta_{i}$ is defined by

$$
\delta_{i}= \begin{cases}1 & \text { for } \quad i=1, \ldots, N_{\text {pin }}, \\ 0 & \text { for } \quad i=\left(N_{\text {pin }}+1\right), \ldots, N,\end{cases}
$$

in which parameter $N_{\text {pin }}$ is the number of pinned nodes, and $q_{i}$ is the control gain associated to node $i$. Without loss of generality, the first $N_{\text {pin }}$ nodes are chosen as the nodes of the network to be pinned. The state vector $\mathbf{x}_{s}\left[t_{0}, \mathbf{x}(0), t\right] \in \mathbb{R}^{n}$, with $\mathbf{x}(0) \in \mathbb{R}^{n}$, is the reference solution, which is assumed to be such that $\dot{\mathbf{x}}_{s}(t)=\mathbf{f}\left[\mathbf{x}_{s}(t), t\right]$ starts from initial conditions that lead to an equilibrium point, a periodic orbit, or a chaotic trajectory.

A more convenient formalism can be introduced. Let us consider a $N$-dimensional Laplacian-like matrix $\Lambda=\left[\Lambda_{i j}\right]$ defined as

$$
\Lambda_{i j}= \begin{cases}-\sigma_{i j} & \text { if }(i, j) \in \mathcal{E}, \\ \sum_{k \in \mathcal{N}_{i}} \sigma_{i k} & \text { if } i=j, \\ 0 & \text { otherwise. }\end{cases}
$$

For this work, it is assumed $\sigma_{i j}=\sigma_{j i}=\sigma$, which is constant for any connection.

Define a $(N+1)$-dimensional extended matrix $\Lambda_{E}$ as

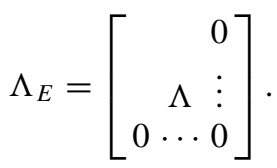

Notice that this extended matrix is also a Laplacian-like matrix. Also, define the pinning matrix as

$$
\Lambda_{\text {pin }}=\left[\begin{array}{cccccccc}
q_{1} & 0 & \cdots & \cdots & \cdots & \cdots & 0 & -q_{1} \\
0 & q_{2} & 0 & \cdots & \cdots & \cdots & 0 & -q^{2} \\
0 & 0 & \ddots & 0 & \cdots & \cdots & 0 & \vdots \\
0 & 0 & 0 & q_{N_{\text {pin }}} & 0 & \cdots & 0 & -q_{N_{\text {pin }}} \\
0 & 0 & 0 & 0 & 0 & \cdots & 0 & 0 \\
\vdots & \vdots & \vdots & \vdots & \vdots & \vdots & \vdots & \vdots \\
0 & 0 & 0 & 0 & 0 & 0 & 0 & 0
\end{array}\right],
$$

which is a $(N+1)$-dimensional Laplacian-like matrix.

Now, omitting the explicit dependence on time $t$ for the sake of brevity, the governing equations of the pinned network (3) can be recast as

$$
\dot{X}=F(X)-\mathbf{H}_{\mathbf{E}} X-\mathbf{H}_{\text {pin }} X, X(0)=X_{0},
$$

in which $X=\left[\mathbf{x}_{1}^{T}, \ldots, \mathbf{x}_{N}^{T}, \mathbf{x}_{s}^{T}\right]^{T} ; \quad F(X)=\left[\mathbf{f}\left(\mathbf{x}_{1}\right)^{T}, \ldots\right.$, $\left.\mathbf{f}\left(\mathbf{x}_{N}\right)^{T}, \mathbf{f}\left(\mathbf{x}_{S}\right)^{T}\right]^{T} ; \mathbf{H}_{\mathbf{E}}=\Lambda_{E} \otimes I_{n}[(N+1) \times n$-dimensional matrix $] ;$ and $\mathbf{H}_{\text {pin }}=\Lambda_{\text {pin }} \otimes I_{n} \quad[(N+1) \times n$-dimensional matrix], with $\otimes$ being the Kronecker product. 


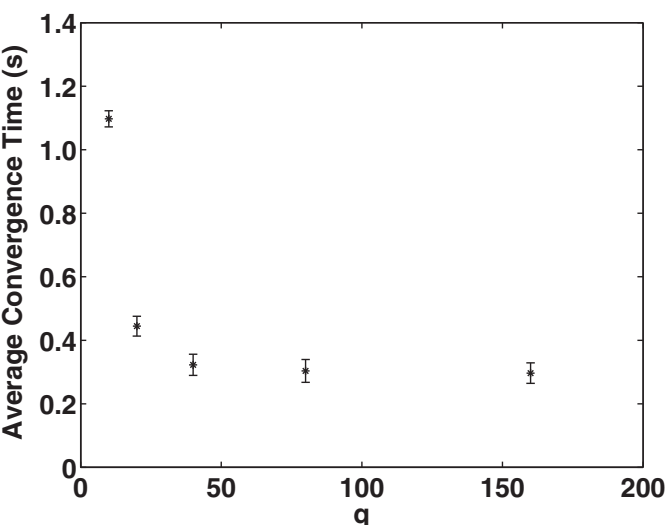

FIG. 2. $q_{i}$ effect: the average synchronization time for different values of control gain $q_{1}$ in a random network with coupling strength $\sigma=10$.

Notice that Eq. (6) can still be written as

$$
\dot{X}=F(X)+\mathbf{G} X, X(0)=X_{0},
$$

in which $M=-\Lambda_{E}-\Lambda_{\text {pin }}$, and $\mathbf{G}=M \otimes I_{n}[(N+1) \times n-$ dimensional matrix]. One can then rewrite Eq. (3) in terms of $M$ coefficients to get the final controlled network equation:

$$
\dot{\mathbf{x}}_{i}=\mathbf{f}\left(\mathbf{x}_{i}\right)-\sum_{j=1}^{N+1} M_{i j} \mathbf{x}_{j}, \quad i=1, \ldots, N+1,
$$

in which $M_{i j}$ is the element $i j$ of matrix $M$.

Matrix $M$ is not always diagonalizable. However, even for this case, the master stability function, which is represented by the variational equations

$$
\dot{\eta}_{h}(t)=\left(D \mathbf{f}-\lambda_{h} I_{n}\right) \boldsymbol{\eta}_{h}(t),
$$

is applicable [12], in which $D \mathbf{f}$ is the Jacobian matrix of $\mathbf{f}$, $\lambda_{h}$ is the $h$ th eigenvalue of matrix $M, \eta_{h}$ represents the $h$ th decoupled node dynamics transverse to the synchronization manifold, and $h=1, \ldots, N$.

In order to quantify the convergence rate to a synchronized state, consider the quantities $W_{h}(t)=\left\|\eta_{h}\right\|^{2}$ (a measure of $\boldsymbol{\eta}_{h}$ boundedness) associated to $\lambda_{h}$. Following the steps in [14], one can get

$$
\dot{W}_{h}(t)=\left[\zeta_{h}(t)-2 \lambda_{h}\right] W_{h}(t),
$$

in which $\zeta_{h}(t)=\frac{\boldsymbol{\eta}_{h}(t)^{T}\left(D \mathbf{f}+D \mathbf{f}^{T}\right) \boldsymbol{\eta}_{h}(t)}{\boldsymbol{\eta}_{h}(t)^{T} \boldsymbol{\eta}_{h}(t)}$.

Considering $\|D \mathbf{f}\| \ll\left\|\boldsymbol{\eta}_{h}\right\|$, with $h=1, \ldots, N$, one can take $D \mathbf{f}+D \mathbf{f}^{T}$ as the fluctuation of $\boldsymbol{\eta}_{h}^{T} \boldsymbol{\eta}_{h}$. The solution for Eq. (10) is approximately given by $W_{h}(t)=W_{h}(0) e^{\left[\int_{0}^{t} \zeta_{h}(t)-2 \lambda_{h} t\right]}$. As such, one concludes that the exponential convergence rate for approaching the synchronized state along the eigenvector $\mathbf{e}_{h}$ associated to the eigenvalue $\lambda_{h}$ is given by $\mu_{h}=-\frac{d \ln W_{h}(t)}{d t}=$ $2 \lambda_{h}$. Therefore, as the convergence speed is restrained by the slowest mode, the convergence rate of the synchronizing process is given by

$$
\mu=\min _{h \neq 1}\left(2 \lambda_{h}\right)=2 \lambda_{\min },
$$

in which $\lambda_{\min }$ is the second lowest eigenvalue of matrix $M$, which is in accordance with the results in [15] (the first lowest eigenvalue is always the zero one).

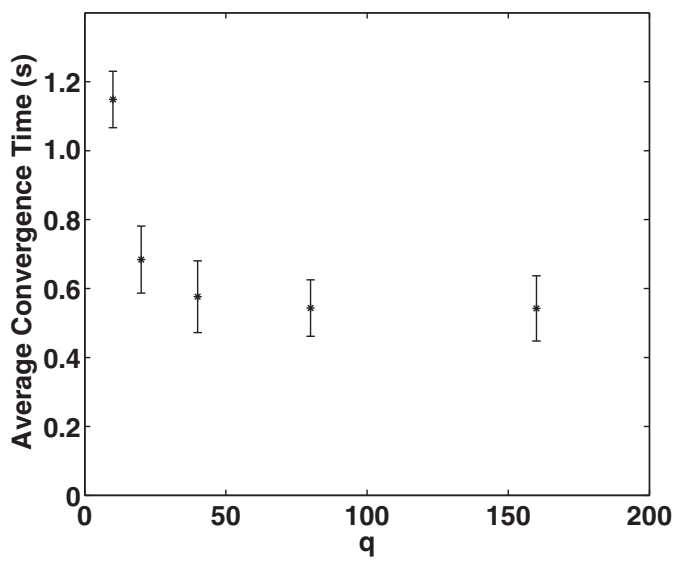

FIG. 3. $q_{i}$ effect: the average synchronization time for different values of control gain $q_{1}$ in a small-world network with coupling strength $\sigma=10$.

From Eq. (11), one can conclude that the convergence rate is directly related to network topology, the coupling strength $\sigma$, and the control gains $q_{i}$. The consequent conclusion is the following: The higher the value of the coupling strength $\sigma$, the higher the convergence rate (by setting $q_{i}=\sigma \tilde{q}_{i} \forall i$, it results in $\mu=2 \sigma \tilde{\lambda}_{\text {min }}$, in which $\left.\tilde{\lambda}_{\text {min }}=\lambda_{\text {min }} / \sigma\right)$. However, it is not so clear how the control gains $q_{i}$ and the number of pinned nodes $N_{\text {pin }}$ influence the convergence rate. Let us address these issues next.

\section{NUMERICAL RESULTS}

In this section, numerical simulations are used to determine the following characteristics regarding pinning-control strategy in networks: (1) the dependence of the convergence rate with the control gains $q_{i}$, (2) the dependence of the convergence rate with the number of pinned nodes $N_{\text {pin }}$, and (3) the relation between the type of node (high degree, low degree, high cluster coefficient, etc.) to be pinned and the synchronization achievement performance.

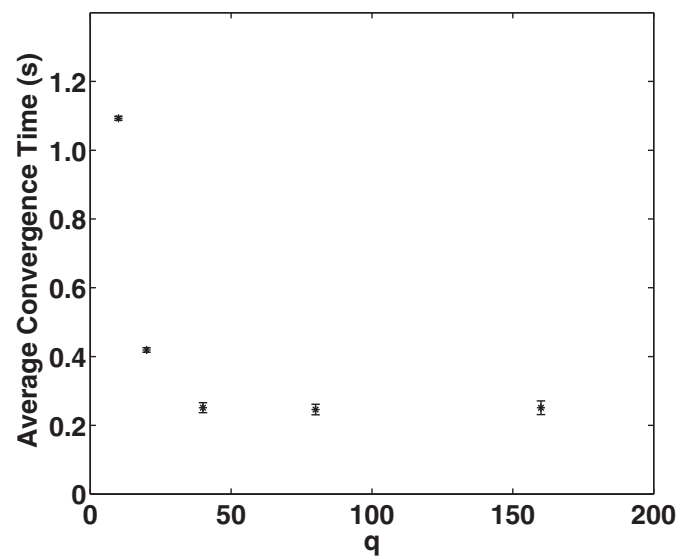

FIG. 4. $q_{i}$ effect: the average synchronization time for different values of control gain $q_{1}$ in a scale-free network with coupling strength $\sigma=10$. 


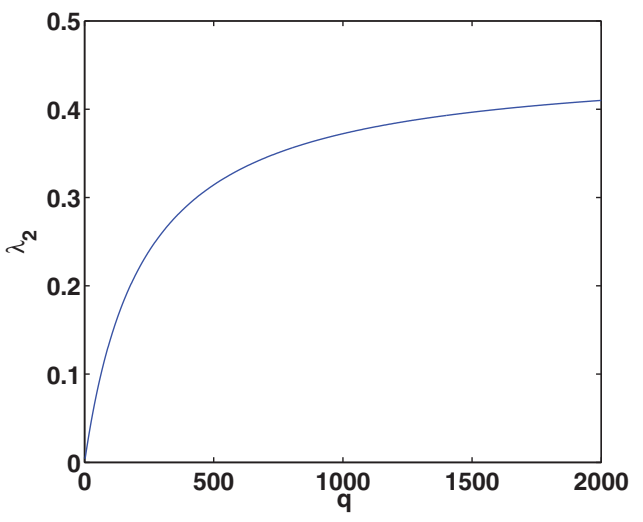

FIG. 5. (Color online) The variation of the $\lambda_{\min }(M)$ value vs control gain $q_{1}$ in a scale-free network.

Consider a controlled network represented by Eq. (3) with 300 identical Chen's oscillators [16] (a variation of Lorenz's system) defined as follows:

$$
\dot{\mathbf{x}}_{i}=\left(\begin{array}{ccc}
-c_{1} & c_{1} & 0 \\
-c_{2} & c_{3} & 0 \\
0 & 0 & -c_{4}
\end{array}\right)\left(\begin{array}{c}
x_{i 1} \\
x_{i 2} \\
x_{i 3}
\end{array}\right)+\left(\begin{array}{c}
0 \\
-x_{i 1} x_{i 3} \\
x_{i 1} x_{i 2}
\end{array}\right),
$$

in which $i=1, \ldots, 300, \quad c_{1}=35, \quad c_{2}=7, \quad c_{3}=28$, and $c_{4}=3$. For these parameters, Chen's system presents chaotic behavior [16]. Initial conditions are randomly chosen for a normal distribution with unitary standard variation, with $x_{1}, x_{2}$, and $x_{3}$ mean values equal to $\sqrt{63}, \sqrt{63}$, and 21 , respectively [16].

In order to check the results, simulations are also carried out using the Rössler oscillator defined as follows:

$$
\dot{\mathbf{x}}_{i}=\left(\begin{array}{ccc}
0 & -1 & -1 \\
1 & d_{1} & 0 \\
0 & 0 & -d_{2}
\end{array}\right)\left(\begin{array}{c}
x_{i 1} \\
x_{i 2} \\
x_{i 3}
\end{array}\right)+\left(\begin{array}{c}
0 \\
0 \\
d_{3}+x_{i 1} x_{i 3}
\end{array}\right)
$$

in which $d_{1}=0.432, d_{2}=4, d_{3}=2$; these are values for which the Rössler oscillator presents chaotic behavior [17]. Initial conditions are randomly chosen for a normal distribution with unitary standard variation, with $x_{1}, x_{2}$, and $x_{3}$ mean values equal to $\sqrt{63}, \sqrt{63}$, and 21 , respectively, and with the reference initial condition set to the fixed point $\mathbf{x}_{s}(0)=\left(0,0, d_{3}\right)$.

In the numerical experiments, three different network types are analyzed: scale-free, small-world, and random networks. The scale-free network topology was generated using the Albert and the Barabàsi [18,19] method with five initial nodes; the small-world network topology was created according to the Watts-Strogatz [19] method with rewiring probability $p_{s w}=0.5$ and starting degree $k_{0}=6$; and the random network topology was randomly generated with an initial wiring probability $p_{r 0}=0.042$. Both scale-free and smallworld topologies present an average node degree $\langle k\rangle=8.0$, while the average node degree for the random topology is $\langle k\rangle=8.08$, which is very close to the first ones. In each simulation, only the initial conditions are changed-network topology is kept fixed.

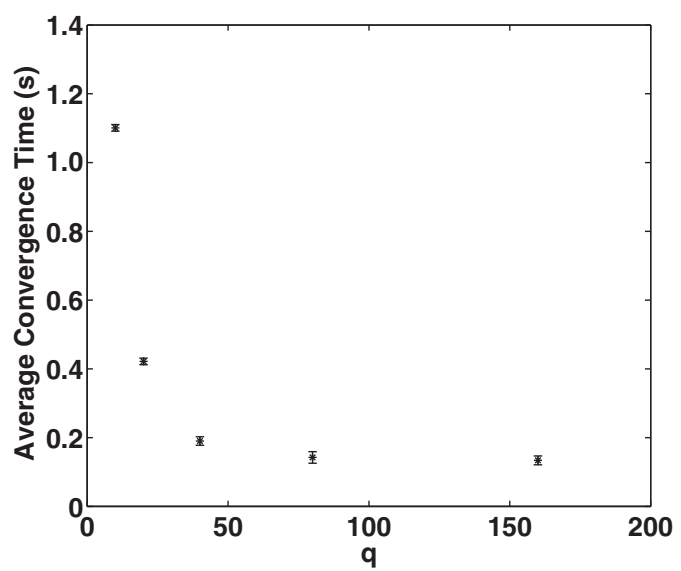

FIG. 6. $q_{i}$ effect with $\sigma=20$ : the average synchronization time for different values of control gain $q_{1}$ in a random network with coupling strength $\sigma=20$.

Finally, let us define now the pinning-control cost by

$$
C=\sigma \sum_{i=1}^{N_{\text {pin }}} q_{i},
$$

in which $q_{i}$ is the control gain associated to node $i, \sigma$ is the network coupling strength, and $N_{\text {pin }}$ is the number of pinned nodes.

\section{A. Control gain $q_{i}$ effect}

First, the effect of increasing control gain value $q_{i}$ on the synchronization time ${ }^{2}$ of the complex network is analyzed. As the stop criteria, the synchronized state is considered to be achieved when $\frac{1}{N+1} \sum_{i=1}^{N+1}\left\|\mathbf{x}_{\mathbf{i}}-\mathbf{x}_{\mathbf{s}}\right\|_{2}<\epsilon=0.001$. In every case, the coupling strength is set to $\sigma=10$, which is a value that guarantees network synchronability. ${ }^{3}$ The number of pinned nodes is set as $N_{\text {pin }}=1$, while the control action is applied to the hub ${ }^{4}$ of each network. Without loss of generality, since the Laplacian matrix can always be rearranged, the hub is set to be node 1 .

Figures 2, 3, and 4 show the average ${ }^{5}$ synchronization time over 50 simulation realizations for different values of control gain $q_{1}$, for the random, small-world, and scale-free networks of Chen's oscillators, respectively. Observe that for all the analyzed networks, the average synchronization time has a

\footnotetext{
${ }^{2}$ Synchronization time can be defined as the time interval from the instant in which the control is on until the instant that we can consider all the nodes of the system are synchronized.

${ }^{3}$ The value of $\sigma$ that guarantees synchronizability can be estimated by applying a method proposed in [16]; estimating the value of $\sigma$ for the three different topologies gives, in the worst case (random topology), $\sigma=77.68$. The estimated value is too conservative, since simulations show that on average over 50 simulation realizations, $\sigma_{A V}=2.03$ guarantees synchronizability of the networks used in our simulations.

${ }^{4} \mathrm{Hub}$ : the hub node of a network is the highest degree node of a network.

${ }^{5}$ In this work, the average corresponds to the mean value over the 50 simulation realizations.
} 


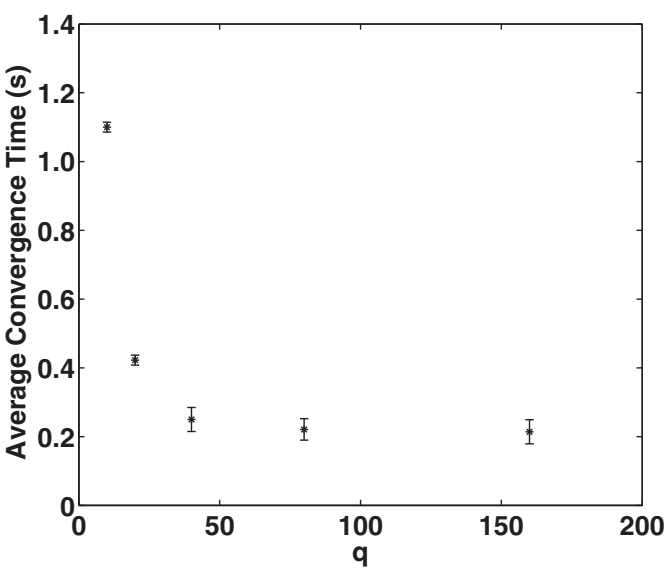

FIG. 7. $q_{i}$ effect with $\sigma=20$ : the average synchronization time for different values of control gain $q_{1}$ in a small-world network with coupling strength $\sigma=20$.

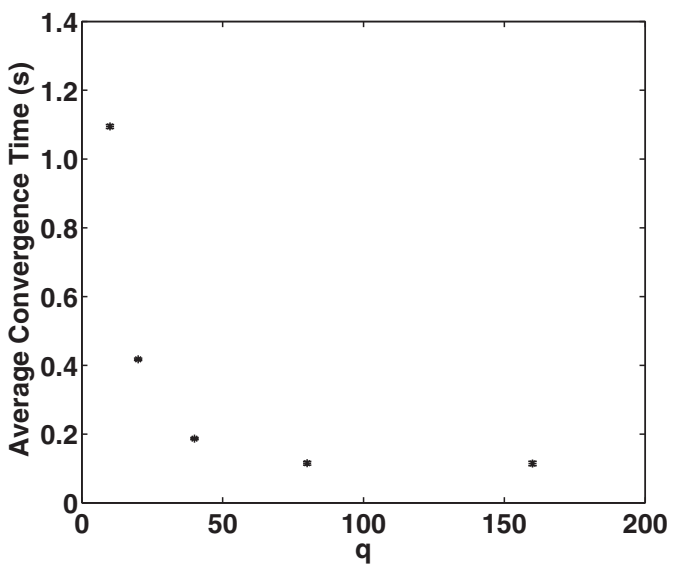

FIG. 8. $q_{i}$ effect with $\sigma=20$ : the average synchronization time for different values of control gain $q_{1}$ in a scale-free network with coupling strength $\sigma=20$.

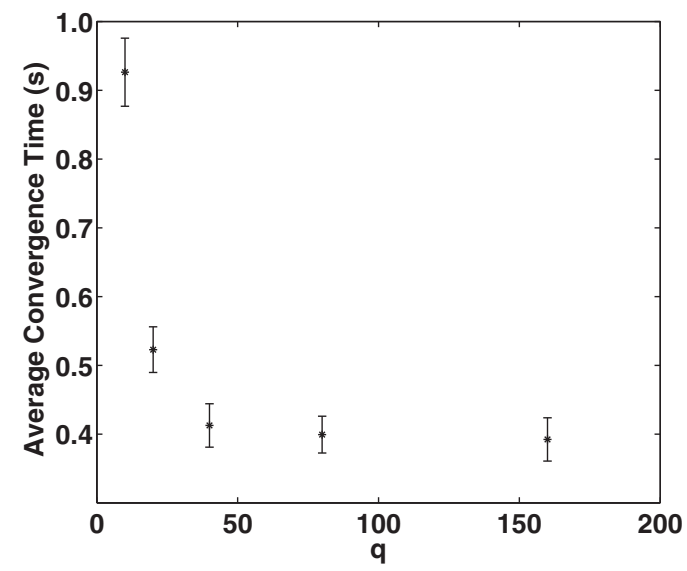

FIG. 9. $q_{i}$ effect with $\sigma=20$ in Rössler network: the average synchronization time for different values of control gain $q_{1}$ in a scale-free network of Rössler oscillators with coupling strength $\sigma=20$.

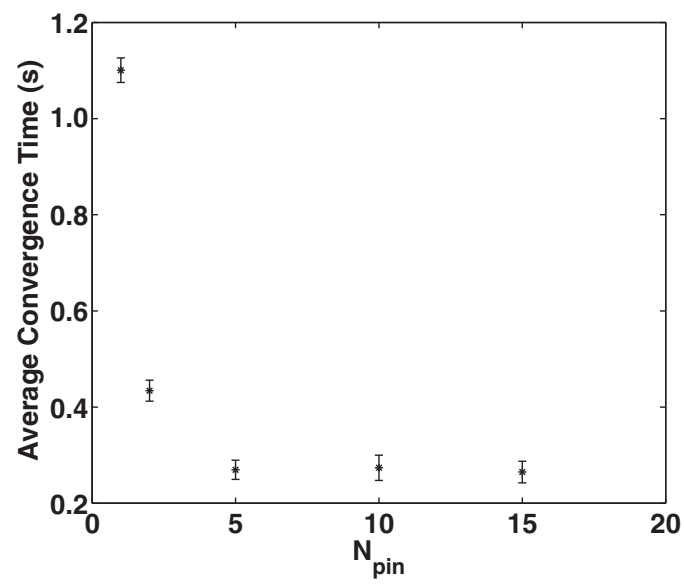

FIG. 10. $N_{\text {pin }}$ effect: the average synchronization time for different number of pinned nodes $N_{\text {pin }}$ in a random network with coupling strength $\sigma=10$ and control gain $q_{1}=10$.

meaningful reduction as $q_{1}$ is varied from $q_{1}=10$ to $q_{1}=20$; however, when $q_{1}$ is varied from $q_{1}=20$ to $q_{1}=40$, and so on, no meaningful reduction of the average synchronization time is verified.

Based on these results, it is possible to make the following conjecture: The increase in $q_{1}$ significantly reduces the synchronization time up to a limit. Beyond this limit, increases in the value of $q_{1}$ may not have any further meaningful effect in synchronization performance. Notice that this observed behavior is in accordance with results obtained in [11], in which it is shown that $\lim _{q_{i} \rightarrow \infty} \lambda_{\min }(M)=c$, in which $c$ is a constant for a given bounded number of pinned nodes $N_{\text {pin }}$. Figure 5 supports this characteristic by showing how $\lambda_{\min }(M)$ [see Eq. (9)] varies with the control gain $q_{1}$ for a scale-free network. Notice that the parameter $\lambda_{\min }$ converges to a constant value, regardless of how high the $q_{1}$ value is. Additionally, the results suggest that this behavior for $\lambda_{\min }(M) \times q_{1}$ is the same for all network topologies. Furthermore, the synchronization time is inversely related to $\sigma$ [see Eq. (11)]. This means that

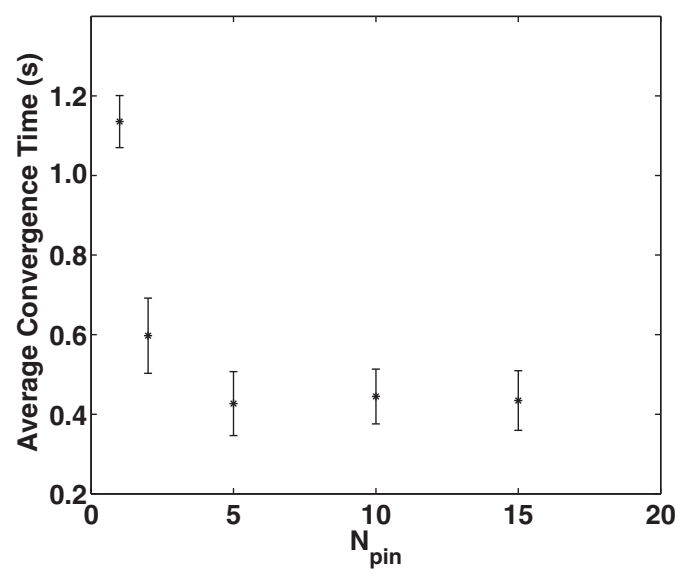

FIG. 11. $N_{\text {pin }}$ effect: the average synchronization time for different number of pinned nodes $N_{\text {pin }}$ in a small-world network with coupling strength $\sigma=10$ and control gain $q_{1}=10$. 


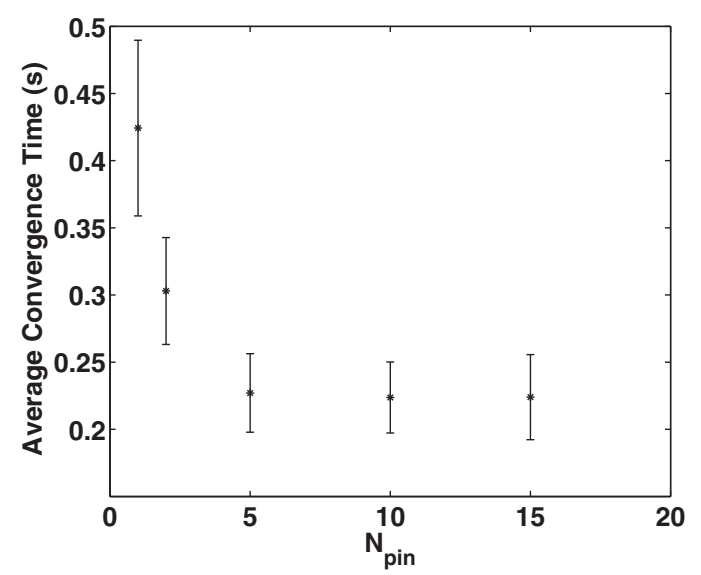

FIG. 12. $N_{\text {pin }}$ effect: the average synchronization time for different number of pinned nodes $N_{\text {pin }}$ in a scale-free network with coupling strength $\sigma=10$ and control gain $q_{1}=10$.

there might exist a nontrivial relationship between an optimum value $q_{i}=q_{i}^{*}$, and the value of $\sigma$.

One can make the last conclusion assuming that in the analysis, $\zeta_{h}(t)$ in Eq. (10) is regarded just as a perturbation. As such, its contribution (which also depends on the initial conditions) to the synchronization time was not quantified.

In order to have some perspective of such relationship, the simulations are repeated, but now setting $\sigma=20$. Figures 6,7, and 8 show the average synchronization time over 50 simulation realizations for different values of the control gain $q_{1}$ for random, small-world, and scale-free networks, respectively.

Figure 9 shows the average synchronization time over 50 simulation realizations for different values of the control gain $q_{1}$ in a scale-free network of Rössler oscillators. Notice that, qualitatively, the results are equivalent to those obtained for Chen's oscillators.

By comparing these results with the previous ones (for $\sigma=10$ ), one can assess that increasing $\sigma$ implies decreasing the synchronization time. As such, by increasing $\sigma$, the same synchronization time can be achieved, but with a smaller value for the gain $q_{1}$. Notice that this numerical behavior is in accordance with the theoretical conclusion presented in Sec. II that states that the network synchronization time is inversely proportional to $\sigma \lambda_{\min }(M)$ [see Eq. (9)].

\section{B. Pinned nodes $N_{\text {pin }}$ effect}

Reference [11] shows that for the limit case where $N_{\text {pin }} \rightarrow$ $N$, in which $N$ is the number of nodes of the network, the value of $\lim _{\substack{q_{i} \rightarrow \infty \\ N \text { in } \rightarrow N}} \lambda_{\min }(M)=c$ increases. This means that the pinning synchronization performance increases. In practice, however, one is able to pin just a bounded number of nodes. In this section, the effect of increasing the number of pinned nodes on the synchronization time is analyzed. For the entire analysis, the parameter values are $\sigma=10, q_{1}=10$. The stop criteria is the same one previously defined in Sec. III A.

\footnotetext{
${ }^{6}$ This value of $q_{i}$ is the one that minimizes both synchronization time and pinning cost, i.e., the $q_{i}$ value such that $\left(\lim _{q_{i} \rightarrow q_{i}^{*}} \lambda_{\min }(m) \simeq c\right)$.
}

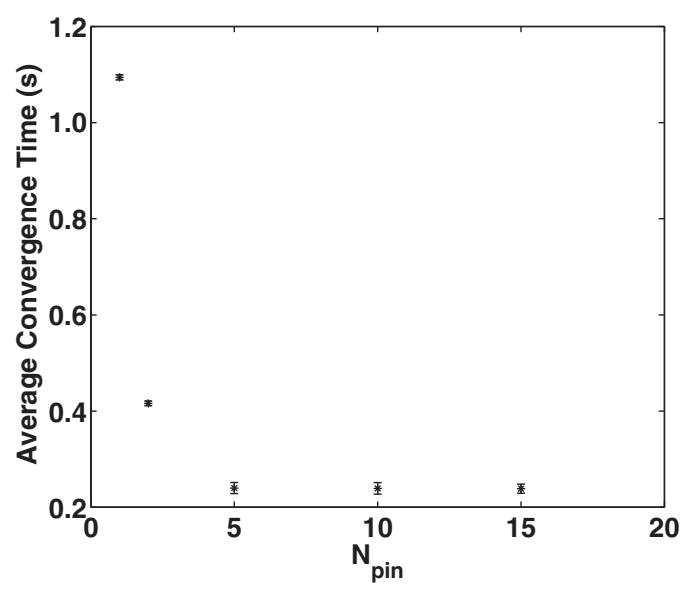

FIG. 13. $N_{\text {pin }}$ effect in Rössler network: the average synchronization time for different number of pinned nodes $N_{\text {pin }}$ in a small-world network of Rössler oscillators with coupling strength $\sigma=10$ and control gain $q_{1}=10$.

The pinned nodes used are the higher degree nodes in each network.

Figures 10, 11, and 12 show the average synchronization time over 50 simulation realizations for different numbers of pinned nodes $N_{\text {pin }}$ for random, small-world, and scale-free networks, respectively. Note that for all of these cases, the average synchronization time decreases when $N_{\text {pin }}$ is varied from $N_{\text {pin }}=1$ to $N_{\text {pin }}=5$. Now, when varying $N_{\text {pin }}$ from $N_{\text {pin }}=5$ to $N_{\text {pin }}=10$, and so on, no meaningful reduction in the average synchronization time occurs. This suggests that there is a critical value for the number of pinned nodes $N_{\text {pin }}^{*}$ that minimizes both synchronization time and pinning cost, i.e., the $N_{\text {pin }}$ value such that $\lim _{\substack{q_{i} \rightarrow \infty \\ N_{\text {pin }} \rightarrow N_{\text {pin }}^{*}}} \lambda_{\text {min }}(M) \simeq c$. This critical number might be somehow related to the coupling strength $\sigma$ and the control gain $q_{i}$.

Figure 13 shows the average synchronization time over 50 simulation realizations for different numbers of pinned nodes $N_{\text {pin }}$ in a small-world network of Rössler oscillators. Notice that, qualitatively, these results are equivalent to the one with a network of Chen's oscillators.

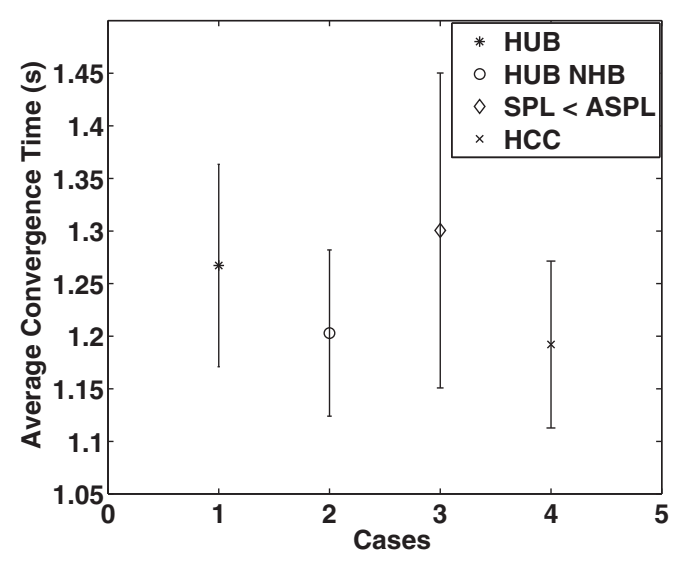

FIG. 14. Node type effect: the average synchronization time for different types of nodes in a random network with $q_{1}=\sigma=10$. 


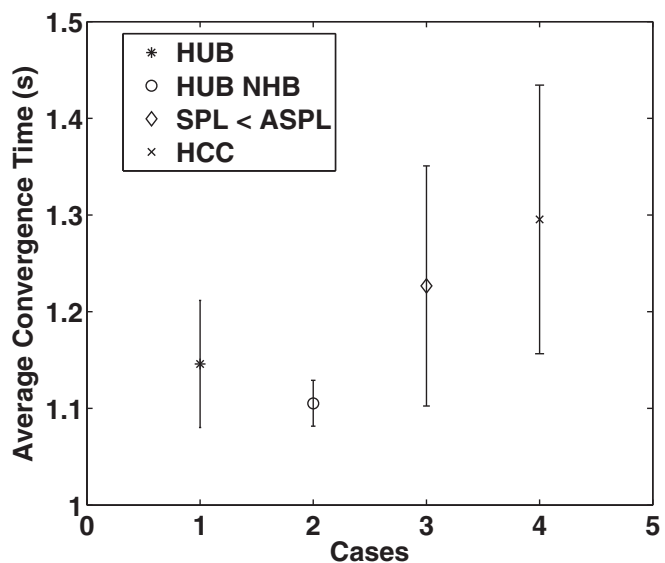

FIG. 15. Node type effect: the average synchronization time for different types of nodes in a small-world network with $q_{1}=\sigma=10$.

\section{Type of node effect}

In Ref. [11], it is shown that for a scale-free network, the pinning synchronization time decreases whenever the nodes to be pinned are changed toward low degree nodes. However, this result was stated for the limit case in which $N_{\text {pin }} \rightarrow N$ and $q_{i} \rightarrow \infty$. In this section, this effect is investigated for bounded values of $N_{\text {pin }}$ and $q_{i}$, and considering random, small-world, and scale-free networks.

The synchronization performance is evaluated based on the average synchronization time of the network for four different types of nodes: (1) hub (labeled HUB in figures), (2) the highest degree node connected to the hub (HUB NHD), (3) a node with $l<l_{a v}$, i.e., a node for which the shortest path length is lower than the average shortest path length [5] of the network (SPL $<$ ASPL), and (4) the highest degree node among the nodes with a higher clustering coefficient [5] (HCC). The stop criteria is the same one defined in Sec. III A. The results can be seen in Figs. 14, 15, and 16, corresponding to random, small-world, and scale-free networks, respectively. The values of parameters $q_{1}$ and $\sigma$ are $q_{1}=\sigma=10$, and there is only one pinned node.

For random and scale-free networks, the shortest average synchronization time is obtained by pinning the highest degree

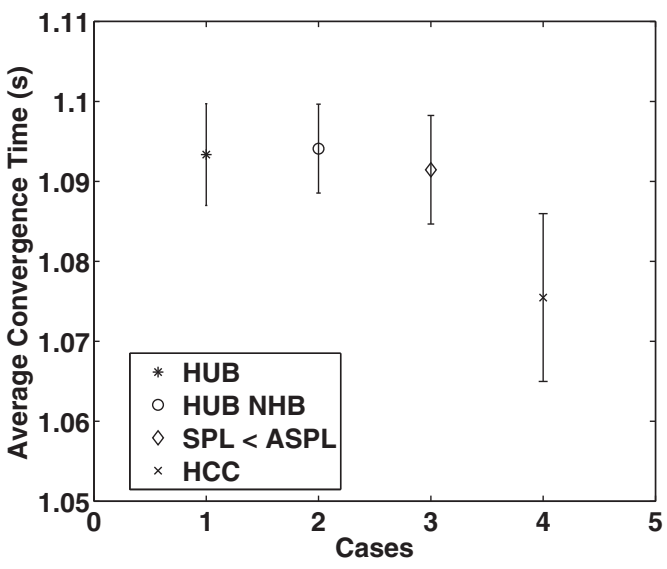

FIG. 16. Node type effect: the average synchronization time for different types of nodes in a scale-free network with $q_{1}=\sigma=10$.

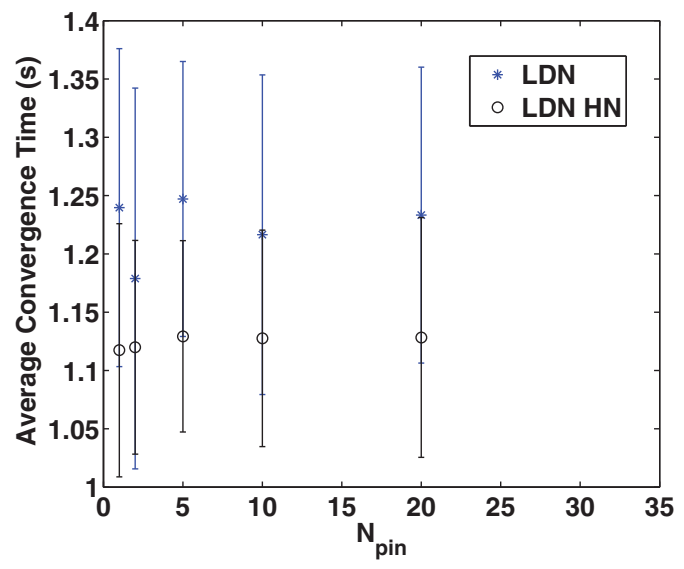

FIG. 17. (Color online) $N_{\text {pin }}$ effect: the average synchronization time for different number of pinned nodes $N_{\text {pin }}$ in a random network with constant pinning-control cost.

node among the nodes with the higher clustering coefficient. For the random network, the degree of the node with the highest clustering coefficient is close to the hub degree. For the scalefree network, however, the highest clustering coefficient node coincides with the network hub.

For the small-world network, the shortest average synchronization time is obtained by pinning the highest degree node connected to the hub, and in second place, by pinning the hub itself.

As such, this investigation suggests that higher degree nodes are a good choice for being pinned because the number of connections among these nodes and the other nodes is higher than in the case where the other types of nodes were considered. Nevertheless, Ref. [10] shows that for regular networks of a few nodes, a better synchronization performance with low pinning cost can be obtained by pinning a high number of nodes adjacent to the hub. In the next section, we investigate the pinning synchronization performance when nodes adjacent to the hub are pinned.

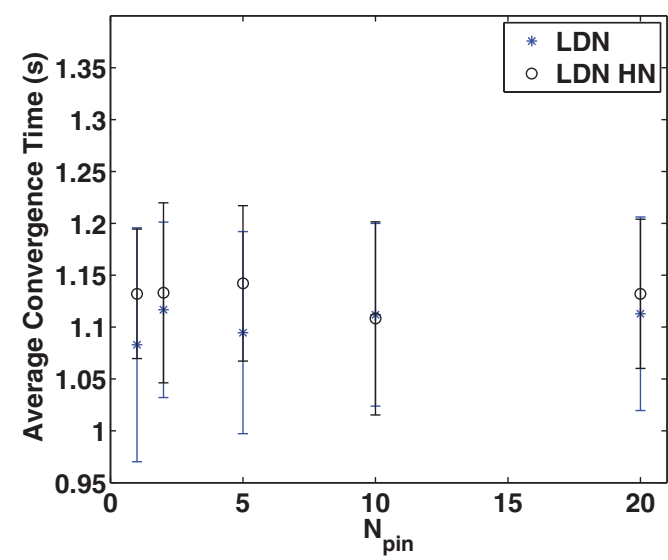

FIG. 18. (Color online) $N_{\text {pin }}$ effect: the average synchronization time for different number of pinned nodes $N_{\text {pin }}$ in a small-world network with constant pinning-control cost. 


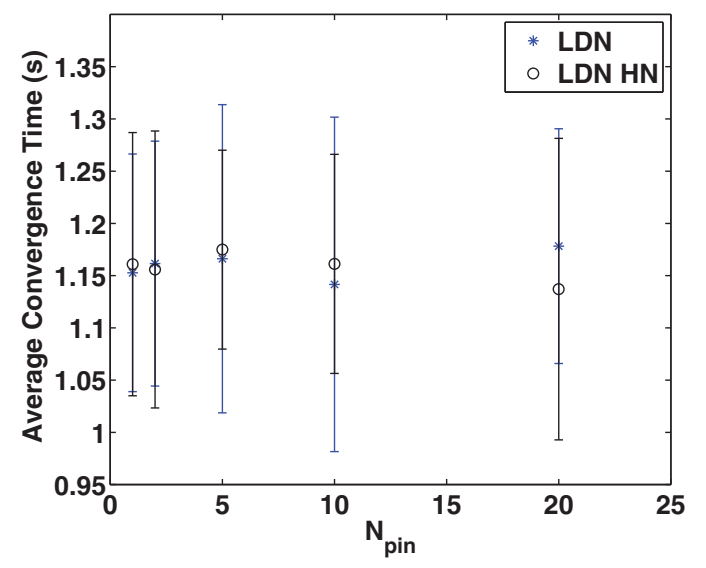

FIG. 19. (Color online) $N_{\text {pin }}$ effect: the average synchronization time for different number of pinned nodes $N_{\text {pin }}$ in a scale-free network with constant pinning-control cost.

\section{Pinning hub neighbors}

In this section, the pinning synchronization performance in the case where lower degree nodes adjacent to the hub (labeled LDN HN in figures) are pinned is investigated. The pinning synchronization performance when lower degree nodes of the network are pinned (LDN) is also investigated.

First, one node adjacent to the hub is pinned and subsequently the number of the pinned nodes $N_{\text {pin }}$ is increased. This is performed by keeping constant the pinning-control cost $C$ [see Eq. (14)] so that $q_{i}=q / N_{\text {pin }}$, where $i=1, \ldots, N_{\text {pin }}$ (for a fixed value of $q$ ). The coupling strength is set to $\sigma=10$ and the control gain to $q=10$.

Figures 17, 18, and 19 show the average synchronization time over 50 simulation realizations for different numbers of pinned nodes $N_{\text {pin }}$ for the random, small-world, and scale-free networks, respectively.

By comparing the results presented in this section with those from the previous one, the conclusion is reached that in the case of scale-free networks, it is better to apply the pinning control to higher degree nodes instead of pinning lower degree nodes adjacent to them. In the case of random and small-world networks, and considering that we are keeping a constant pinning-control cost, our results indicate that pinning lower degree nodes adjacent to the hub (or even pinning lower degree nodes of the network) could lead to a better performance than pinning the network hub. One might expect, however, that when $N_{\text {pin }} \gg 1$ and even keeping a constant control cost, nodes adjacent to higher degree nodes would be a good choice to be pinned.

\section{CONCLUSION}

In this work, we investigated the effect of the network coupling strength $\sigma$, the control gain $q_{i}$, the number of pinned nodes $N_{\text {pin }}$, and the type of the pinned node on the performance of pinning synchronization control.

The results indicate that increasing the control gain $q_{i}$ and/or the number of pinned nodes $N_{\text {pin }}$ does not guarantee a meaningful reduction of the synchronization time. The reason for this is that the nodes' dynamics also contributes to the final synchronization time. The results allow us to conjecture that there may exist an optimum set of values $q_{i}$ and $N_{\text {pin }}$ that guarantees an optimum synchronization performance with minimum pinning-control cost. Moreover, we also assess that this set of values $q_{i}$ and $N_{\text {pin }}$ is related to the coupling strength $\sigma$ and the network topology.

The relation between node type and pinning synchronization performance is also investigated. It was observed that in most cases, high degree nodes are a good choice for being pinned. However, when $N_{\text {pin }} \gg 1$, nodes adjacent to the highest degree node are also a good choice to be pinned, independently of the network topology.

\section{ACKNOWLEDGMENT}

The authors would like to thank FAPESP and CNPq for financial support of this work.
[1] P. Lu and Y. Yang, Int. J. Control 83, 364 (2010).

[2] J. Aljadeff, N. Levine-Small, Z. Yekutieli, and L. Meshulam, in Dynamics Days South America 2010: International Conference on Chaos and Nonlinear Dynamics (São José dos Campos, 2010).

[3] W. Wang, I. Z. Kiss, and J. L. Hudson, Chaos 10, 248 (2010).

[4] B. Ballobàs, Random Graphs, London Mathematical Society Monographs (Academic, London, 1985).

[5] X. F. Wang and G. Chen, IEEE Circuits Syst. 3, 6 (2003).

[6] L. M. Pecora and T. L. Carroll, Phys. Rev. Lett. 64, 821 (1990).

[7] R. O. Grigoriev, M. C. Cross, and H. G. Schuster, Phys. Rev. Lett. 79, 2795 (1997).

[8] F. Sorrentino, M. di Bernardo, F. Garofalo, and G. Chen, Phys. Rev. E 75, 046103 (2007).
[9] M. Porfiri and M. di Bernardo, Automatica 44, 3100 (2008).

[10] L. Rong, D. Zhi-Sheng, and G. Chen, Chin. Phys. B 18, 106 (2009).

[11] Y. Zou and G. Chen, Physica A 388, 2931 (2009).

[12] T. Nishikawa and A. E. Motter, Physica D 224, 77 (2006).

[13] L. M. Pecora and T. L. Carroll, Phys. Rev. Lett. 80, 2109 (1998).

[14] G. Yan, G. Chen, J. Lü, and Z.-Q. Fu, Phys. Rev. E 80, 056116 (2009).

[15] C. W. Wu, Phys. Lett. A 319, 495 (2003).

[16] J. Zhou, J. A. Lu, and J. Lü, Automatica 44, 996 (2008).

[17] O. E. Rössler, Phys. Lett. A 57, 397 (1976).

[18] A.-L. Barabási and R. Albert, Science 286, 509 (1999).

[19] D. J. Watts and S. H. Strogatz, Nature (London) 393, 440 (1998). 